\title{
Modelagem matemática das perdas de água e solo em latossolo argiloso sob sistemas
}

\section{de manejo}

\author{
Mathematical modeling of soil and water losses in Oxisol under management systems \\ Modelización matemática de pérdidas de suelo y agua en arcillosos Oxisol bajo sistemas de manejo
}

\section{Resumo}

Este trabalho teve por objetivo modelar matematicamente as perdas de água e solo em um Latossolo argiloso com diferentes sistemas de manejo, por meio dos atributos físicos de solo e da cobertura vegetal. A área experimental localizou-se no município de Santa Tereza do Oeste - PR, em lavoura de soja. Os tratamentos consistiram em três sistemas de manejo: sistema plantio direto tradicional, sistema plantio direto gessado, sistema plantio direto com escarificação e doze tratamentos de sistema plantio direto com espécies de cobertura em consórcio ou não, denominadas "recuperadoras" de estrutura do solo. Os dados de perdas de água e solo foram coletados a cada precipitação nas calhas coletoras instaladas em cada tratamento ao longo do ciclo da soja. O delineamento foi inteiramente casualizado. Foi utilizado o software Statistica para o ajuste de curva das perdas de água e solo e foram gerados submodelos para estimar valores da palha e cobertura verde ao longo do ciclo da soja. De modo geral, as maiores perdas estão relacionadas com maior densidade e menor macroporosidade do solo, enquanto a relação entre a massa seca da palha e a cobertura verde foi inversamente proporcional. A utilização de função definida por partes para modelar matematicamente foi adequada para estimar valores de cobertura verde ao longo do ciclo da soja.

Palavras-chave: Erosão hídrica; Modelos; Otimização.

\begin{abstract}
The objective of this work was to mathematically model the losses of water and soil in a Oxisol with different management systems, through the physical attributes of soil and vegetation cover. The experimental area was located in the municipality of Santa Tereza do Oeste - PR, in a soybean field. The treatments consisted of three management systems: traditional no-tillage system, plastered no-tillage system, no-tillage system with scarification and twelve treatments of no-tillage system with cover species in intercropping or not, called "recoverers" of soil structure. Data on water and soil losses were collected at each precipitation in the collector gutters installed in each treatment throughout the soybean cycle. The design was completely randomized. The Statistica software was used to adjust the curve of water and soil losses and submodels were generated to estimate straw and green cover values along the soybean cycle. In general, the highest losses are related to higher density and lower soil macroporosity, while the relationship between straw dry mass and green cover was inversely proportional. The use of part-defined function to mathematically model was adequate to estimate green cover values along the soybean cycle.
\end{abstract}

Keywords: Water erosion; Models; Optimization. 


\begin{abstract}
Resumen
El objetivo de este trabajo fue modelar matemáticamente las pérdidas de agua y suelo en un Oxisol arcilloso con diferentes sistemas de manejo, a través de los atributos físicos del suelo y la cobertura vegetal. El área experimental se ubicó en el municipio de Santa Tereza do Oeste - PR, en un campo de soja. Los tratamientos consistieron en tres sistemas de manejo: sistema de labranza cero tradicional, sistema de labranza cero enyesado, sistema de labranza cero con escarificación y doce tratamientos del sistema de labranza cero con especies de cobertura en cultivos intercalados o no, denominados "recuperadores" de estructura del suelo. Los datos sobre pérdidas de agua y suelo se recolectaron en cada precipitación en las canaletas colectoras instaladas en cada tratamiento a lo largo del ciclo de la soja. El diseño fue completamente aleatorio. Se utilizó el software Statistica para ajustar la curva de pérdidas de agua y suelo y se generaron submodelos para estimar los valores de cobertura verde y paja a lo largo del ciclo de la soja. En general, las mayores pérdidas están relacionadas con una mayor densidad y una menor macroporosidad del suelo, mientras que la relación entre la masa seca de paja y la cobertura verde fue inversamente proporcional. El uso de la función parcialmente definida para modelar matemáticamente fue adecuado para estimar los valores de cobertura verde a lo largo del ciclo de la soja. Palabras clave: Erosión hídrica; Modelos; Optimización.
\end{abstract}

\title{
1. Introdução
}

Apesar dos benefícios do Sistema Plantio Direto (SPD) como a rotação de culturas e conservação dos resíduos vegetais sobre a superfície do solo, a prática correta ainda é pouco utilizada nas lavouras brasileiras. Como consequência, a compactação do solo, as perdas de água, solo e nutrientes, o aumento do custo de produção e a quebra da estabilidade da produtividade estão afetando as áreas com SPD em razão do desconhecimento dos produtores ou a simplificação de procedimentos (Dias, 2014).

A partir da baixa mobilização no solo que o SPD propõe, a compactação do solo ocorre em maior extensão e intensidade nas camadas superficiais (Nascimento et al., 2016). Com isso, as chuvas intensas e abundantes da região Sul do Brasil e o não respeito à condição de umidade no momento de colheita e tratos culturais agravam ainda mais as circunstâncias.

Pandey et al. (2016) realizaram uma revisão sobre os modelos mecanísticos de predição de perdas de solo por erosão, mostrando que os principais problemas na aplicação dos modelos são decorrentes da complexidade natural, precisão e os requisitos de dados para a entrada. Mello et al. (2016), afirmam que as limitações destacadas foram que os modelos não capturam aspectos relevantes, como os impactos das plantas de cobertura no processo da erosão. Vanwalleghem et al. (2017) também afirmaram que, muitas vezes, não são consideradas em modelos de erosão as propriedades dinâmicas que afetam a erodibilidade do solo e a topografia.

Além disso, os artigos de revisão mostram que a maioria dos modelos são testados em bacias agrícolas situadas apenas em países desenvolvidos, o que exige antes da aplicação dos mesmos, calibração específica do local (Pandey et al., 2016). Sendo assim, muitos desafios foram transcorridos pelos cientistas com o passar do tempo para modelar os sistemas naturais e os modelos são a forma de melhor custo-benefício para prever os impactos das ações antrópicas sobre a natureza (Mello et al., 2016).

Em razão da grande diversidade dos modelos, sempre existe uma versão para se resolver qualquer problema prático, assim como um mesmo modelo pode ser utilizado para resolver diversos problemas (Pandey et al., 2016). Dentre as questões que podem ser gerenciadas com os modelos, são destacados os impactos extremos do clima nas inundações, deslizamentos de terras, secas, problemas com a colheita, entre outros (Mello et al., 2016).

A principal contribuição às estratégias do uso da terra e água nas lavouras para se precaver da erosão é advinda dos modelos matemáticos. Por meio dos resultados das simulações, os profissionais da área agrícola conseguem compreender melhor os impactos gerados pela mudança de manejo ou remoção de espécies (Mello et al., 2016). Quanto às previsões de dados, a modelagem e a simulação computacional tornam-se, na realidade, a única ferramenta disponível (Vanwalleghem et al, 2017).

Olivetti et al. (2015) realizaram a modelagem, com o objetivo de estimar as taxas de erosão hídrica nos Latossolos da sub-bacia hidrográfica do Sul do estado de Minas Gerais empregando o RUSLE (Revised Universal Soil Loss Equation), em conjunto com técnicas de geoestatística e sistemas de informação geográfica. Os autores afirmaram que a modelagem foi relevante para auxiliar na determinação de medidas de conservação e recuperação do solo, além de estimar as perdas de solo e 
identificar as áreas mais suscetíveis à erosão.

Com o objetivo de comparar as estimativas de perdas de solo feitas em modelo WEPP (Water Erosion Prediction Project) e USLE (Equação Universal de Perda do Solo), Gvozdenovich et al. (2017) utilizaram parcelas de coleta de escoamento superficial localizadas na Argentina, com milho. Os autores evidenciaram a supremacia do modelo WEPP sobre o USLE referente aos quatro tratamentos, por ter apresentado a estimativa mais eficiente.

Em uma microbacia agrícola sob SPD no sul do Brasil, Didoné, Minella e Evrard (2017) avaliaram o impacto de diferentes medidas de conservação de solo por meio do modelo WaTEM/SEDEM (Water and Tillage Erosion Model and Sediment Delivery Model) para simular a erosão do solo. Os autores observaram que as simulações do modelo indicaram baixo impacto favorável do plantio direto sobre a erosão. Eles consideraram essencial tomar medidas complementares para aumentar a cobertura vegetal para reduzir significativamente as perdas de solos nessas áreas rurais.

Diante do exposto, este trabalho teve por objetivo modelar matematicamente as perdas de água e solo em um Latossolo argiloso com diferentes sistemas de manejo, por meio dos atributos físicos de solo e da cobertura vegetal.

\section{Metodologia}

O estudo é uma pesquisa experimental de caráter quantitativo por meio de coletas de campo e laboratorial, com análise de dados numéricos (Pereira et al., 2018).

\section{1 Área experimental}

O trabalho foi realizado na área experimental do Instituto Agronômico do Paraná (IAPAR) polo regional de Santa Tereza do Oeste - PR. O relevo local é suavemente ondulado, com declividade variando de 0,21 a 5,41\%. O solo é classificado como Latossolo Vermelho Distroférrico típico, com textura argilosa a muito argilosa (EMBRAPA, 2018). As coletas foram realizadas durante a safra 2017/2018 com a cultura de soja sobre a palhada de crambe em SPD.

\subsection{Tratamentos e delineamento experimental}

Em 12 parcelas foram implantadas espécies de cobertura vegetal após o cultivo da soja, sendo 6 de verão e 6 de inverno e três sistemas sistemas de manejo (plantio direto tradicional (testemunha), plantio direto gessado e plantio direto escarificado). Os quinze primeiros tratamentos são apresentados na Tabela 1.

Como o estudo foi aproveitado de um experimento de longa duração, foi observado em pesquisas anteriores que o solo é considerado de alta qualidade, resultando em tratamentos homogêneos. Assim, foi elaborada uma faixa compactada para todos os tratamentos, exceto T5, por meio do rolo compactador para diversificar os valores dos parâmetros físicos do solo e aumentar o repertório de dados para a modelagem estatística. Totalizando, dessa forma, 29 tratamentos (compactados e não-compactados). 
Tabela 1. Quinze primeiros tratamentos do experimento com as espécies "recuperadoras” de estrutura implantadas após o cultivo da soja.

\begin{tabular}{ll}
\hline Notação & Tratamentos \\
\hline \multicolumn{2}{c}{ Espécies de cobertura/consórcios de verão } \\
\hline T1 & Milheto \\
T3 & Guandu anão \\
T8 & Crotalaria juncea \\
T9 & Feijão guandu \\
T10 & Crotalaria spectbilis \\
T13 & Mucuna preta \\
\hline Espécies de cobertura/consórcios de inverno \\
\hline T2 & Aveia branca \\
T4 & Aveia preta \\
T6 & Centeio \\
T7 & Aveia preta + nabo forrageiro \\
T12 & Aveia preta + tremoço branco \\
T14 & Aveia preta + ervilha forrageira \\
\hline Sistemas de uso e manejo \\
\hline T5 & Sistema plantio direto com escarificação até 0,3 m de profundidade \\
T11 & Sistema plantio direto com aplicação de 3 t ha ${ }^{-1}$ de gesso em superfície \\
T15 & Sistema plantio direto tradicional (Testemunha) \\
\hline
\end{tabular}

Fonte: Autores (2021).

Foram construídas 29 calhas coletoras, uma em cada tratamento, com as áreas delimitadas em $9 \mathrm{~m}^{2}(3,0 \times 3,0 \mathrm{~m})$ por separadores de grama conforme ilustrado na Figura 1 e foram monitoradas as perdas de água e solo ocorridas durante as chuvas naturais. As calhas são de material PVC de 3 metros de comprimento e 0,1 m de diâmetro, alocadas no sentido da declividade do solo de modo que a água escoada possa alcançar até os recipientes de 25 litros (bombonas plásticas) em um nível abaixo da superfície. Os recipientes foram conectados às calhas por meio de mangueiras corrugadas de 3/4”.

Figura 1. Modelo das calhas coletoras e suas medidas.

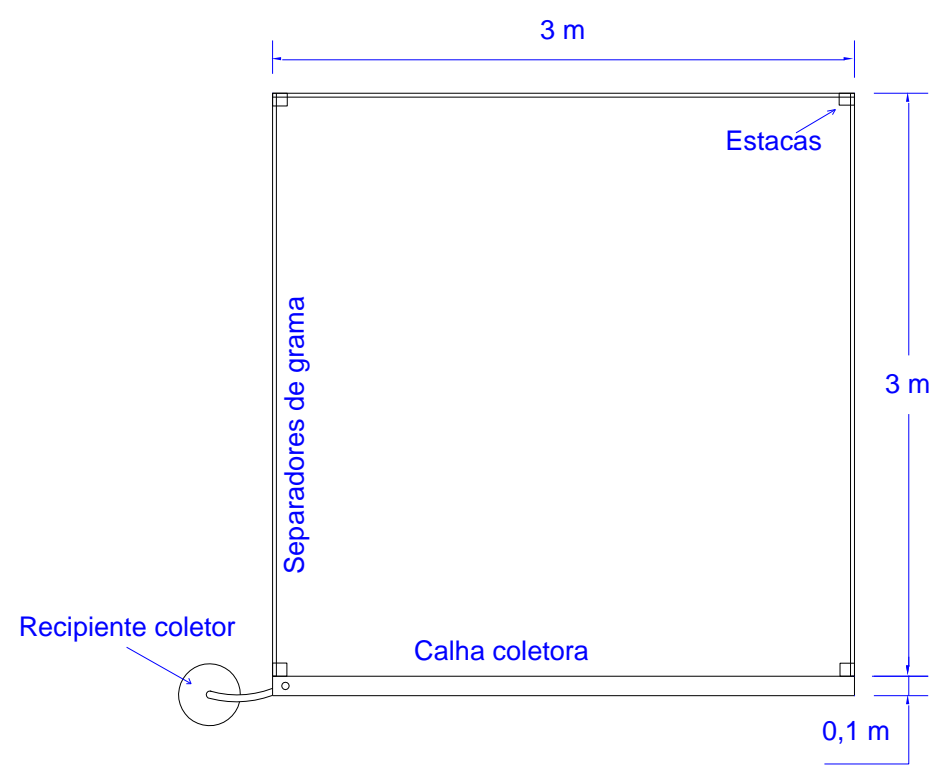

Fonte: Autores (2021).

O delineamento experimental foi inteiramente ao acaso, sendo os 29 tratamentos incluindo as faixas compactadas e os 20 eventos sucedidos de precipitações ao longo do ciclo da soja representando as repetições. 


\subsection{Determinação das propriedades físicas}

Antes da semeadura da soja e após a sua colheita, foram coletadas, em dois pontos por cada tratamento, as amostras de solo indeformadas em três camadas de solo (0-10, 10-20 e 20-30 cm). No Laboratório de Física do Solo (LAFIS) da Universidade Estadual do Oeste do Paraná, Campus Cascavel, foi avaliada a densidade do solo, a macro, micro e a porosidade total. A granulometria foi determinada pelo método da pipeta em dispersão em água e em hidróxido de sódio. A condutividade hidráulica do solo saturado foi calculada utilizando o permeâmetro de carga constante, conforme metodologia preconizada por Claessen (1997).

\subsection{Coleta de água e solo}

Ao todo, foram realizadas 20 coletas sucedidas entre os dias 08/11/2017 a 10/02/2018 (

Figura 2), fixando-se um horário do dia para coletar. O conteúdo armazenado nas bombonas foram homogeneizados e quantificados por meio de provetas graduadas. Além disso, a cada parcela de $9,3 \mathrm{~m}^{2}$ - considerando os $0,3 \mathrm{~m}^{2}$ da calha de PVC - foi retirado $500 \mathrm{~mL}$ do conteúdo das bombonas em garrafas pet, sendo que $50 \mathrm{~mL}$ foram destinados à quantificação do solo no LAFIS.

Figura 2. Precipitação acumulada durante os dias da coleta.

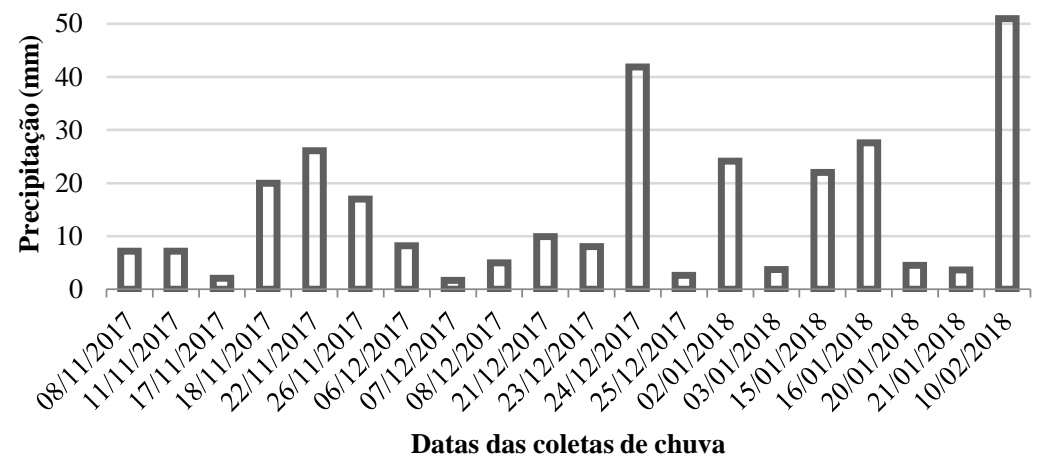

Fonte: Autores (2021).

Para quantificar as perdas de solo, foram retiradas subamostras de $50 \mathrm{~mL}$ homogeneizadas e colocadas na estufa a $105^{\circ} \mathrm{C}$ para evaporação da água e que restasse apenas a massa seca do solo. Por meio disso, foram estimadas as perdas equivalentes para 1 hectare, utilizando das conversões de unidades e medidas.

\subsection{Coleta de palha e cobertura verde}

No caso da coleta de palha, feita com periodicidade de 15 dias, foi jogada aleatoriamente uma armação quadrangular de 30x30 cm na parcela experimental. Após, foi coletada toda a palha no interior da armação, fazendo-se duas repetições por parcela. O material foi colhido em sacos de papel kraft identificados. Por fim, foram pesadas as amostras de palha após colocadas na estufa a $65^{\circ} \mathrm{C}$ até massa constante.

Sobre a coleta da cobertura verde, foram escolhidos dois pontos aleatórios por parcela para se colocar em cima da cobertura foliar da soja uma armação quadrangular de $1 \mathrm{~m}^{2}$. Por meio de um bastão para câmera, foi tirada uma foto de modo que o retrato enquadrasse toda a armação. Assim, tal foto foi recortada digitalmente para, em seguida, passar por uma leitura do 
software Canopeo (Canopeo APP, 2018), o qual processa valores em porcentagem referentes à cobertura vegetal, captando apenas a coloração verde da área coletada.

\subsection{Análise estatística}

Para a análise dos dados, empregou-se ao software R Studio (R Core Team, 2015) para determinar a média, mediana, variância, coeficiente de variação, coeficiente de assimetria, coeficiente de curtose e análise de variância.

\subsection{Modelagem matemática}

Foram gerados modelos de regressão que relacionaram o comportamento de variáveis de interesse aos estudos e, especificamente, àquelas que mais influenciam na perda de água e solo, tais como densidade do solo $(D s)$, macroporosidade (Macro), massa seca da palha (Msp) e cobertura verde ( $\mathrm{Cob}$ ). Como a intensidade de chuva afetou significativamente os valores das perdas, foi considerada como a variável mediadora para todos os parâmetros.

O software STATISTICA (Statsoft, 2011) foi utilizado para a geração dos ajustes à modelagem e seus respectivos gráficos. No processo de ajuste de curvas, foi utilizado o Método de Levenberg-Marquardt, implementado no software de otimização para o Problema dos Quadrados Mínimos não Linear.

Assim sendo, os modelos da perda de água (P.agua) foram ajustados por uma função polinomial de grau 1 de duas variáveis do tipo:

$$
\text { P.agua }(\operatorname{Int}, f)=a \operatorname{Int}+b f+c
$$

em que Int é a intensidade máxima de chuva para cada evento de precipitação, $f$ os parâmetros físicos conhecidos mediante as observações em campo a serem considerados na modelagem (densidade do solo, macroporosidade, cobertura verde e massa seca da palha), enquanto $a, b$ e $c$ são constantes definidas por meio de ajustes.

Já em relação aos modelos da perda de solo (P.solo), a função que melhor se ajustaram aos dados observados foi uma exponencial do tipo:

$$
\text { P. solo }(\text { Int }, f)=a \cdot \exp (b \cdot \operatorname{Int})+c \cdot f+d
$$

em que $P$ é a função referente às perdas e $a, b, c$ e $d$ as constantes obtidas por meio de ajustes.

A respeito da massa seca da palha $(M s p)$, foram estimados valores para cada dia de coleta de chuva por meio da construção de submodelos. Por conseguinte, foi estabelecido para cada data da coleta de palha, a quantidade de dias que se passou após a semeadura da soja.

Após isso, por meio do Método dos Quadrados Mínimos, foram ajustados os valores das coletas à uma função polinomial de grau 3 do tipo:

$$
M s p(t)=a t^{3}+b t^{2}+c t+d
$$

em que $t$ representa a quantidade de dias que se passou após a semeadura da soja. Foi utilizado polinômio de grau 3 pois foi ajustado adequadamente aos dados, considerando-se a métrica do coeficiente de determinação $\left(R^{2}\right)$, e mostrou uma tendência representativa em função do tempo.

Sobre a cobertura verde, apresentada em forma de porcentagem, também foram estimados valores para cada dia de coleta. As condições inicial e final para a geração dos modelos podem ser representadas como:

$$
\operatorname{Cob}(0)=\operatorname{Cob}(135)=0
$$

em que $\operatorname{Cob}(t)$ representa a porcentagem de cobertura verde ao longo do tempo $t$. Indicando que no dia da semeadura e no dia em que apresentou a secagem completa das folhas de soja (135 dias após a semeadura), respectivamente, a cobertura verde da soja é equivalente a $0 \%$. A despeito dessas restrições, foi observado que nenhum polinômio satisfez à condição física de: 


$$
0 \leq \operatorname{Cob}(t) \leq 100
$$

Isto é, todos os graus de polinômio, ou apresentavam predições com valores negativos, ou com valores muito acima de $100 \%$.

Portanto, o método escolhido, que foi verificado ser mais próximo aos dados, foi a utilização de uma função definida por partes como:

$$
\operatorname{Cob}_{\tau}(t)= \begin{cases}\sum_{i=0}^{n_{1}} a_{i} t^{i}, & t<40 \\ \sum_{i=0}^{n_{2}} b_{i} t^{i}, & t \geq 40\end{cases}
$$

sendo $\tau$ o número do tratamento, $n_{1}$ o grau do polinômio referente ao modelo antes do $40^{\circ}$ dia, $a_{i}$ e $b_{i}$ os parâmetros ajustados aos dados e $n_{2}$ o grau do polinômio referente ao modelo depois do $40^{\circ}$ dia. Dessa forma, apresentaria uma tendência antes dos 40 dias, com uma curva representando um crescimento gradual da cobertura e uma outra tendência para depois dos 40 dias, com uma curva representando a estabilidade da cobertura com o tempo e seu decrescimento.

\section{Resultados e Discussão}

\subsection{Estatística descritiva}

A Tabela 2 apresenta a estatística descritiva das perdas de água e solo para todos os tratamentos e dias de coleta. As médias representam, portanto, as perdas aproximadas para cada tratamento e coleta de chuva, podendo estimar as perdas acumuladas para um determinado tempo multiplicando os valores médios pelo tempo.

Tabela 2. Estatística descritiva das perdas de água e solo.

\begin{tabular}{ccc}
\hline & Água (L/ha) & Solo (kg/ha) \\
\hline Mín. & 107,50 & 0,02 \\
Q1 & 2151,00 & 0,54 \\
Q2 & 4086,00 & 1,603 \\
Méd. & 7247,00 & 7,882 \\
Q3 & 10220,00 & 6,115 \\
Máx. & 26880,00 & 205,3 \\
S & 7292,26 & 20,33 \\
S & 53176984 & 413,26 \\
CV (\%) & 100,63 & 257,93 \\
Ass. & 1,30 & 0,93 \\
K & 0,19 & 0,17 \\
ANOVA (p-valor) & 0,9998 & 0,59 \\
\hline
\end{tabular}

Mín: mínimo; Q1: $1^{\circ}$ quartil; Q2: $2^{\circ}$ quartil ou mediana; Méd.: média; Q3: $3^{\circ}$ quartil; Máx.: máximo; S: desvio padrão; S²: variância; CV: coeficiente de variação; Ass.: assimetria; K: curtose; ANOVA: análise de variância. Fonte: Autores (2021).

Observando a tabela, pode-se perceber que o coeficiente de variação para todas as perdas foi elevado, mostrando a sua heterogeneidade por ter utilizado os dias da coleta como repetição. Visto que a estatística descritiva apresentada na tabela é referente aos dados de todas as coletas de chuva, que passaram por intensidades desde baixas até altas. Pelos p-valores da análise de variância, como foram acima de 0,05 , não foram identificadas diferenças significativas nas perdas de água e solo, o que pode ser justificado pela boa estrutura de solo que a área experimental apresenta. 


\subsection{Submodelos}

Para gerar os modelos das perdas de água e solo, em função da massa seca da palha e da cobertura verde, foi necessário estimar os valores para cada coleta de chuva. Não foi observado diferença significativa entre os tratamentos pela análise de variância a $5 \%$ de significância.

\subsubsection{Massa seca da palha ao longo do ciclo da soja}

Como citado na Equação (3), a função polinomial utilizada foi a de grau 3. A Figura 3 mostra um exemplo de submodelo com um alto coeficiente de determinação.

Figura 3. Exemplo de submodelo da massa seca da palha ao longo do ciclo da soja. Os dados observados são representados pelos pontos, enquanto a linha de tendência é o resultado da equação mostrada acima.

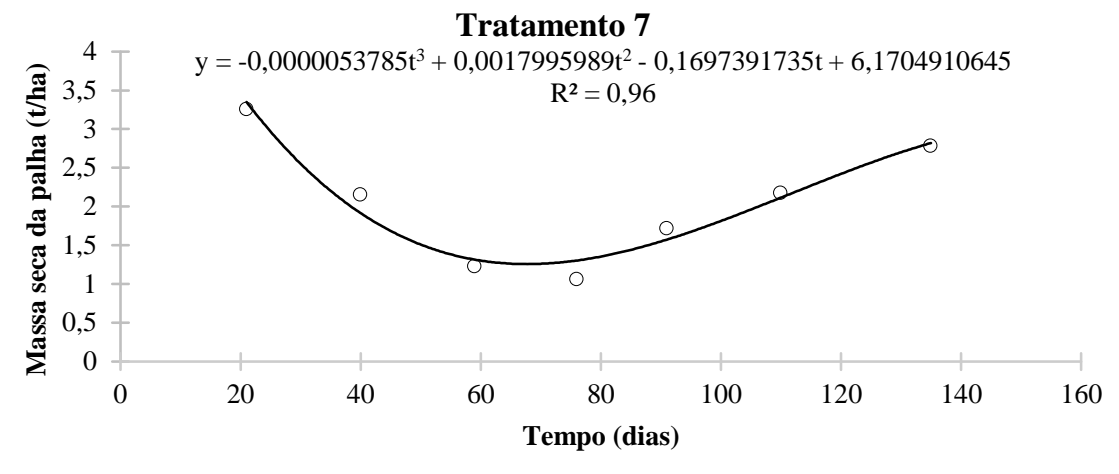

Fonte: Autores (2021).

Além disso, para todos os submodelos calculados, a curva representou de forma plausível o comportamento da massa seca da palha ao longo do ciclo da soja, visto que nos primeiros dias que sucedem a semeadura, o solo é coberto pelos resíduos da cultura anterior (crambe). Assim, com o passar do tempo, a palha vai se decompondo, diminuindo a sua massa consequentemente. Após isso, com a proximidade dos últimos estágios vegetativos da soja, os resíduos que cobrem o solo passam a ser da própria soja e aumentam a proporção até a maturação plena.

\subsubsection{Cobertura verde ao longo do ciclo da soja}

Para a geração dos submodelos da cobertura verde ao longo do ciclo da soja, foi necessário definir uma função geral em que os ajustes devessem cumprir as condições de restrições da Equação (5). Isto é, o limite inferior deveria ser 0\% e o limite superior $100 \%$ para representar valores mais próximos do real. 
Figura 4. Exemplo de submodelo para cobertura verde. (a) função polinomial de grau 3 para os primeiros 40 dias; (b) função polinomial de grau 6 para depois dos 40 dias e (c) função definida por partes com a junção das duas funções.

(a)

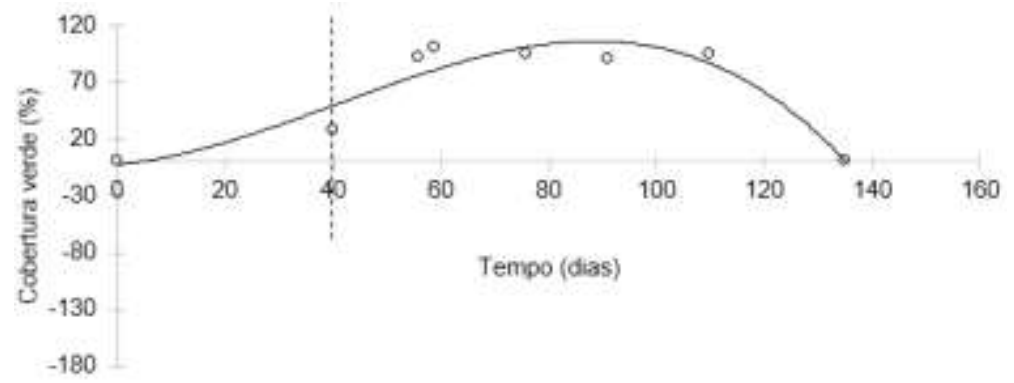

(b)

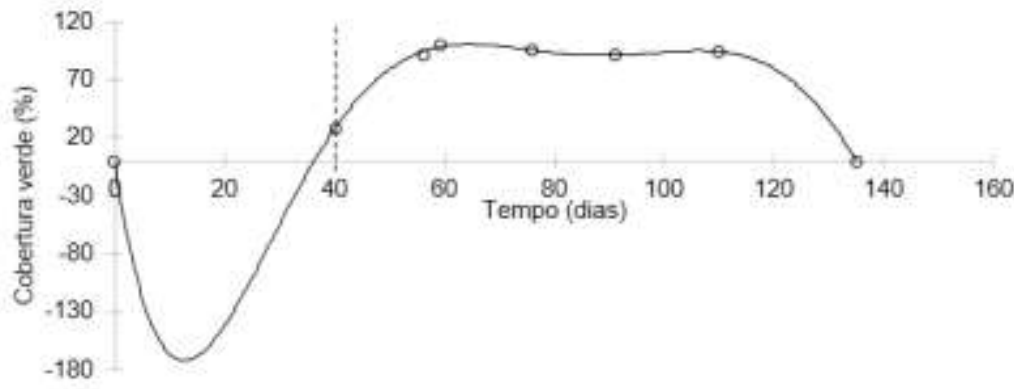

(c)

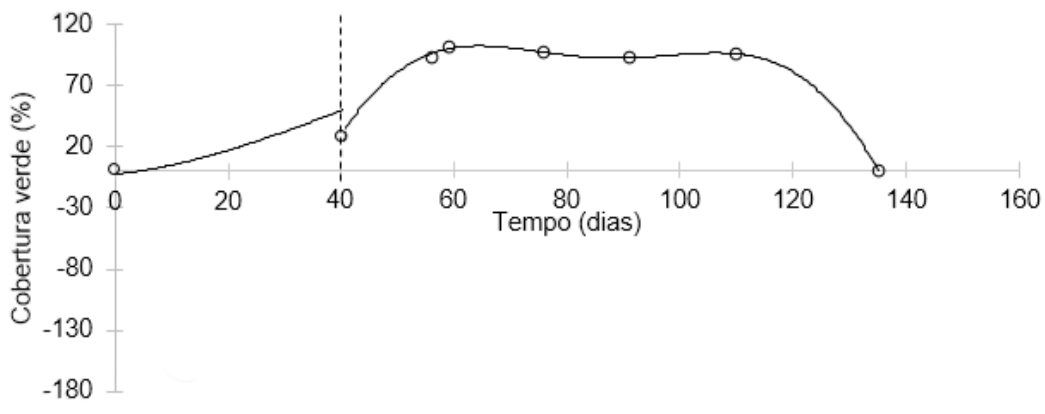

Fonte: Autores (2021)

As curvas geradas pelos submodelos também são de fácil interpretação, visto que o tamanho das folhas de soja vai crescendo ao longo do tempo, alcança uma estabilidade em determinados dias, até chegar à mudança rápida de coloração das folhas e estas se desprenderem dos caules, tornando-se uma matéria morta sobre o solo.

\subsection{Modelos}

\subsubsection{Perda de água}

Os modelos gerados para a perda de água são apresentados na Tabela 3. Os baixos coeficientes de determinação são associados pela alta variabilidade dos dados coletados, como foi mostrado na Tabela 2, além da coleta ser a campo, não sendo possível controlar diversos outros fatores que podem influenciar nas perdas. 
Tabela 3. Modelos de perda de água em função da intensidade máxima de chuva por evento de precipitação $\left(\mathrm{mm} \mathrm{h}^{-1}\right)$, densidade do solo $\left(\mathrm{g} \mathrm{cm}^{-3}\right)$, macroporosidade $(\%)$, massa seca da palha $\left(\mathrm{t} \mathrm{ha}^{-1}\right)$ e cobertura vegetal $(\%)$.

\begin{tabular}{|c|c|}
\hline Equações & $\mathbf{R}^{2}$ \\
\hline P.agua $($ Int,$D s)=767,982 \operatorname{Int}+1754,54 D s-210,99$ & 0,4353 \\
\hline$P . \operatorname{agua}($ Int, Mac $)=767,982$ Int $-117,75$ Mac $+3303,48$ & 0,4358 \\
\hline P.agua $(\operatorname{Int}, M s p)=859,505 \operatorname{Int}+344,611 M s p+11,3519$ & 0,5935 \\
\hline P. agua $($ Int,$C o b)=858,818 \operatorname{Int}-12,044 \operatorname{Cob}+1517,64$ & 0,5952 \\
\hline
\end{tabular}

P. agua: perda de água; Int: intensidade máxima de chuva por evento de precipitação; $D s$ : densidade do solo; Mac: macroporosidade; $M s p$ : massa seca da palha e Cob: cobertura verde. Fonte: Autores (2021).

O comportamento da faixa de tendência ilustrado na Figura 5 evidenciou que houve aumento da perda de água com a intensidade de chuva, resultado semelhante foi encontrado por Oliveira et al. (2015) ao constatarem a correlação linear e positiva entre a precipitação e as perdas de água por escoamento superficial.

Figura 5. Perda de água em função da (a) densidade do solo, (b) macroporosidade, (c) massa seca da palha e (d) cobertura verde.

(a)

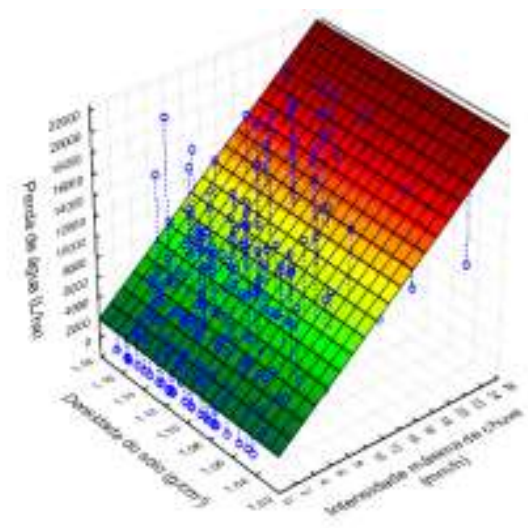

(c)

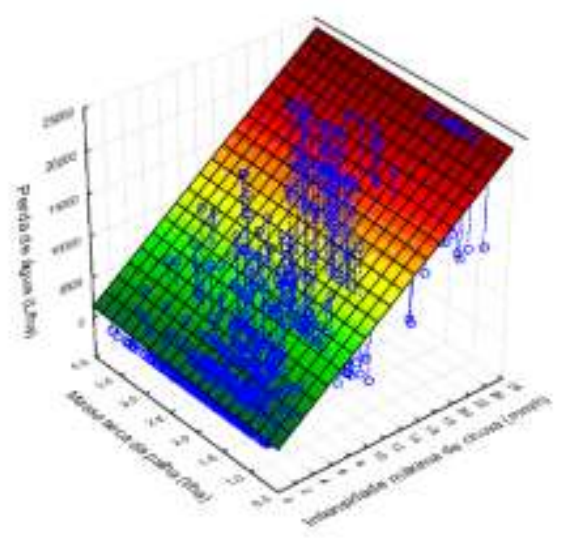

(b)

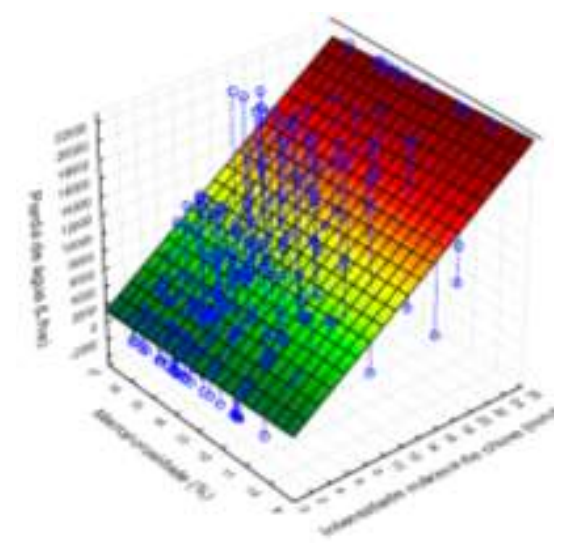

(d)

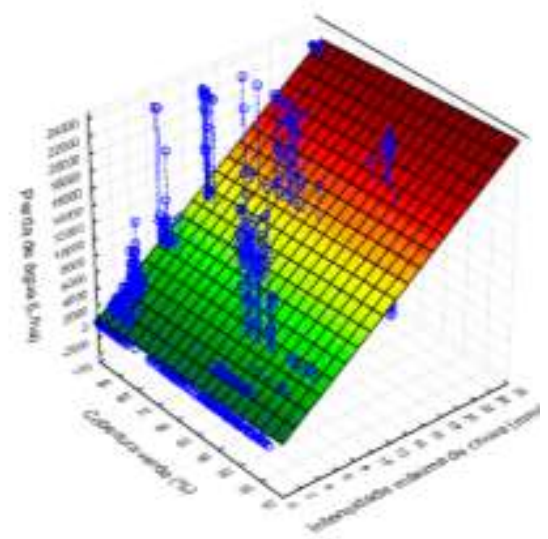

Fonte: Autores (2021). 
Foi possível observar um pequeno aumento da perda de água com a elevação da densidade (Figura 4a), assim, a compactação do solo pode acarretar maiores perdas. Já em função da macroporosidade (Figura 4b), houve aumento da perda de água com a redução do volume de macroporos, visto que, com menos poros é dificultada a infiltração de água no solo, aumentando o escoamento superficial.

Os resultados corroboram com o trabalho de Cândido et al. (2014) quando observaram, ao avaliar a influência dos atributos físicos do solo na erosão hídrica, que houve correlação positiva acerca da densidade do solo e correlação negativa na macroporosidade.

No tocante à massa seca da palha (Figura 4c), observou-se a elevação das perdas de água com a aumento da massa seca da palha. O que contraria Prosdocimi et al. (2016) quando observaram, ao avaliar o efeito da palha de cevada no escoamento superficial, que as perdas de água e solo reduziram com a utilização da palha.

Portanto, esse efeito deve ser analisado pelo tempo e a influência da cobertura verde das plantas de soja, como foi possível verificar na Figura 4. Vale salientar que, na época de maior massa seca da palha sobre o solo (Figura 3), foi quando se identificou a menor cobertura verde e vice-versa. Além disso, as equações apresentadas na Tabela 3 mostraram que as perdas de água em função da massa seca da palha e da cobertura verde obtiveram valores próximos de coeficiente de determinação $(0,5935$ e 0,5952 , respectivamente) e do coeficiente $a$ (859,505 e 858,818, respectivamente). Ademais, os coeficientes $b$ salientaram sinais inversos $(+344,61$ e $-12,04$, respectivamente). Com isso, torna-se evidente que essas relações são inversamente proporcionais.

Já em relação à cobertura verde ilustrada na Figura 4 d, de forma isolada, as perdas de água foram menores em maiores porcentagens de cobertura, concordando com Carvalho et al. (2015) quando afirmam que a maior cobertura do solo, por desenvolvimento natural da colheita, reduz as perdas de água e solo. Almeida et al. (2016) também observaram que o tratamento mais suscetível ao processo erosivo foi na condição em que a cobertura vegetal foi igual a zero.

Sendo assim, nas épocas de menor porcentagem de cobertura verde, também manifestam maior massa seca da palha. Indicando, dessa maneira, o sentido físico quanto ao comportamento do ajuste da perda de água em função da massa seca da palha.

\subsubsection{Perda de solo}

Os modelos gerados para a perda de solo são destacados na Tabela 4. A função utilizada foi uma exponencial, como visto na Equação (2). A Figura 5a mostra o principal motivo de se usar uma exponencial em vez de uma linear, evidenciando que esta função alcança valores de perda próximos a zero. Além disso, a tendência mostrou que as maiores perdas estão associadas aos maiores valores de densidade, isso para intensidades de chuva mais elevadas.

Tabela 4. Modelos de perda de solo em função da intensidade máxima de chuva por evento de precipitação $\left(\mathrm{mm} \mathrm{h}^{-1}\right)$, densidade do solo $\left(\mathrm{g} \mathrm{cm}^{-3}\right)$, macroporosidade $(\%)$, massa seca da palha $\left(\mathrm{t} \mathrm{ha}^{-1}\right)$ e cobertura vegetal $(\%)$.

\begin{tabular}{cc}
\hline Equações & $\mathbf{R}^{\mathbf{2}}$ \\
\hline P. solo $($ Int,$D s)=11,926 \exp (0,060853$ Int $)+10,1374$ Ds $-23,3$ & 0,2462 \\
P. solo $($ Int,$M a c)=11,8806 \exp (0,060967$ Int $)-0,50257$ Mac $-5,3174$ & 0,2473 \\
P. solo $($ Int,$M s p)=11,5209 \exp (0,061868$ Int $)-0,24766$ Msp $-11,16$ & 0,2944 \\
P. solo $($ Int, Cob $)=10,5836 \exp (0,06481$ Int $)-0,03853$ Cob $-7,606$ & 0,2991 \\
\hline
\end{tabular}

Int: intensidade máxima de chuva por evento de precipitação; Ds: densidade do solo; Mac: macroporosidade; Msp: massa seca da palha; Cob: cobertura verde. Fonte: Autores (2021). 
Nas perdas em função da macroporosidade, mostradas na Figura 5b, foi observada a redução da perda de solo com o aumento do volume dos poros, para chuvas mais elevadas. Como a maior macroporosidade está associada à maior capacidade de infiltração, as perdas de solo por escoamento são reduzidas.

Figura 6. Perda de solo em função da (a) densidade do solo, (b) macroporosidade, (c) massa seca da palha e (d) cobertura verde.

(a)

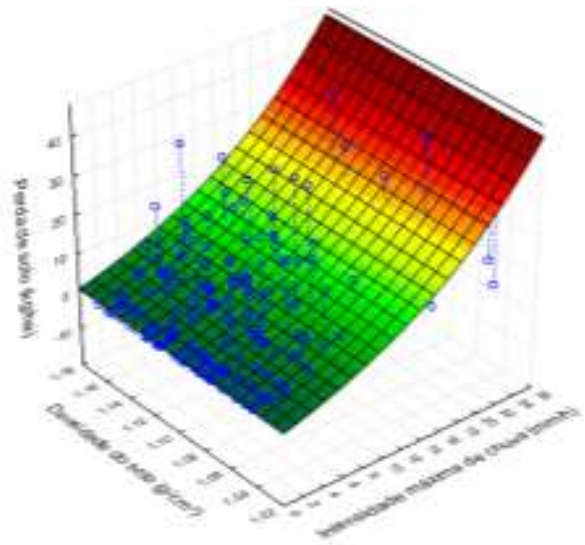

(c)

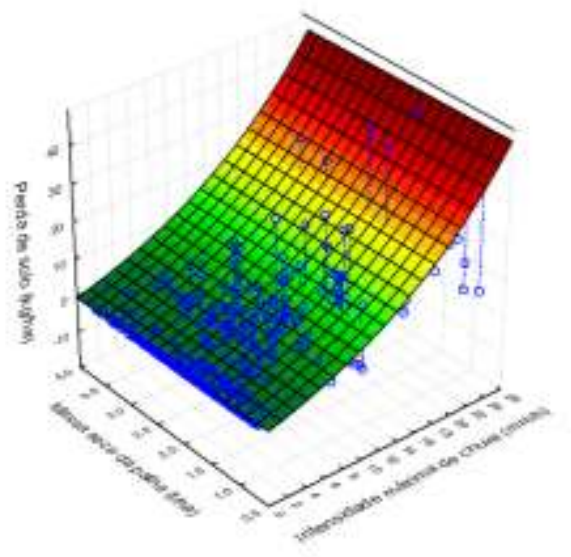

(b)

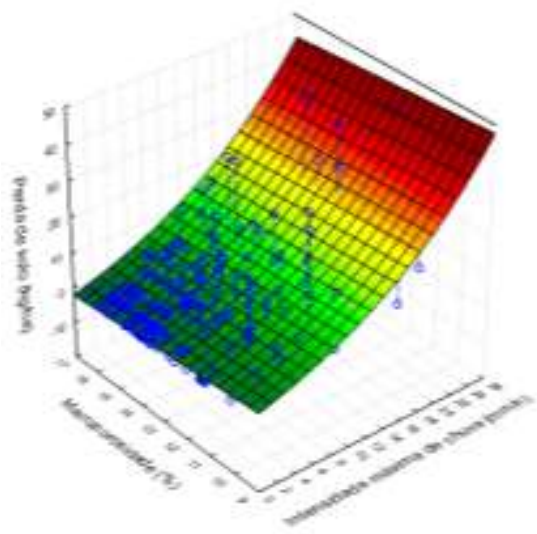

(d)

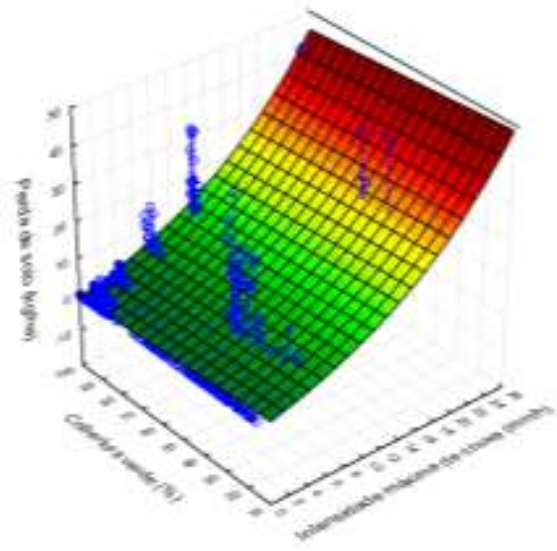

Fonte: Autores (2021).

A Figura 5 (c) mostra a relação das perdas com a massa seca da palha. Diferente da perda de água, esta não apresentou proporção muito clara aparentemente, pois as perdas se mantiveram constantes em diferentes níveis de palha. No entanto, ao analisar os sinais dos coeficientes do modelo (Tabela 4), a tendência mostrou redução das perdas de solo com o aumento da massa seca da palha. Já as perdas em relação à cobertura verde (Figura 5d), assim como ocorreu com as de água, apresentaram redução com o aumento da cobertura.

No trabalho de Volk e Cogo (2014), a partir do qual avaliaram as perdas de solo e água por erosão por meio de chuva simulada de intensidade de chuva de $64 \mathrm{~mm} \mathrm{~h}^{-1}$, utilizaram resíduos culturais de milho e observaram que houve diminuição na perda total de solo, tendendo ao valor nulo. O modelo gerado pelos autores também foi uma função exponencial, apresentado como: 


$$
\text { P. } \operatorname{solo}(\mathrm{Cob})=7,1532 \exp (-0,59 \mathrm{Cob})
$$

com $\mathrm{R}^{2}$ equivalente a 0,7532 e $C o b$ em porcentagem de palha coberta no solo.

Ramos et al. (2014) utilizaram no estudo da influência das condições de superfície na erosão hídrica, modelos exponenciais e concluíram que as perdas de solo reduziram de modo considerável com o aumento da cobertura do solo e da rugosidade superficial. O mesmo também foi realizado por Bertol et al. (2014) durante um projeto de 10 anos.

\section{Conclusão}

De modo geral, as maiores perdas de água e solo estão relacionadas com maior densidade e menor macroporosidade do solo. A relação entre a massa seca da palha e a cobertura verde foi inversamente proporcional, mostrando que a combinação entre palha e cobertura verde controlou a erosão ao longo do ciclo. A função definida por partes se mostrou satisfatório para estimar valores de cobertura verde ao longo do ciclo de desenvolvimento da soja. Uma sugestão para trabalhos futuros é de se utilizar a Regressão Linear Múltipla com todas as variáveis, além de observar o grau de correlação linear de Pearson entre as variáveis.

\section{Agradecimentos}

Os autores agradecem à Fundação Araucária pelo auxílio financeiro e ao Instituto Agronômico do Paraná pelo apoio técnico-logístico.

\section{Referências}

Adviento-Borbe, M. A. A., Barnes, B. D., Iseyemi, O., Mann, A. M., Reba, M. L., Robertson, W. J., ... \& Teague, T. G. (2018). Water quality of surface runoff and lint yield in cotton under furrow irrigation in Northeast Arkansas. Science of the Total Environment, 613, 81-87.

Almeida, W. S. D., Carvalho, D. F. D., Panachuki, E., Valim, W. C., Rodrigues, S. A., \& Varella, C. A. A. (2016). Erosão hídrica em diferentes sistemas de cultivo e níveis de cobertura do solo. Pesquisa Agropecuária Brasileira, 51, 1110-1119.

Bertol, I., Ramos, R. R., Barbosa, F. T., González, A. P., Ramos, J. C., \& Bandeira, D. H. (2013). Water erosion in no-tillage monoculture and intercropped systems along contour lines. Revista Brasileira de Ciência do Solo, 37, 521-528.

Bertol, I., Barbosa, F. T., Mafra, Á. L., \& Flores, M. C. (2014). Soil water erosion under different cultivation systems and different fertilization rates and forms over 10 years. Revista Brasileira de Ciência do Solo, 38(6), 521-528.

Cândido, B. M., Silva, M. L. N., Curi, N., \& Batista, P. V. G. (2014). Erosão hídrica pós-plantio em florestas de eucalipto na bacia do rio Paraná, no leste do Mato Grosso do Sul. Revista Brasileira de Ciência do Solo, 38, 1565-1575.

Canopeo APP. (2018). Canopeo Web. http://canopeoapp.com

Carvalho, D. F. D., Eduardo, E. N., Almeida, W. S. D., Santos, L. A., \& Alves, T. (2015). Water erosion and soil water infiltration in different stages of corn development and tillage systems. Revista Brasileira de Engenharia Agrícola e Ambiental, 19, 1072-1078.

Claessen, M. E. C. (1997). Manual de métodos de análise de solo. Embrapa Solos-Documentos (INFOTECA-E).

Corsato, L. G., Quiessi, J. A., Kanno, O. Y., Penteado, R. B., Arabori, R. M., Belorte, L. C. C., \& Lima, M. E. D. (2016). Diagnóstico e reabilitação agroambiental de trecho de bacia hidrográfica por sensoriamento remoto e turbidez da água. Pesquisa Agropecuária Brasileira, 51, $1099-1109$.

Dechen, S. C. F., Telles, T. S., Guimarães, M. D. F., \& Maria, I. C. D. (2015). Perdas e custos associados à erosão hídrica em função de taxas de cobertura do solo. Bragantia, 74, 224-233.

Dias, C. Simplificação do Plantio Direto reduz eficiência da lavoura. https://www.embrapa.br/busca-de-noticias/-/noticia/1909275/simplificacao-do-plantiodireto-reduz-eficiencia-da-lavoura

Didoné, E. J., Minella, J. P. G., \& Evrard, O. (2017). Measuring and modelling soil erosion and sediment yields in a large cultivated catchment under no-till of Southern Brazil. Soil and Tillage Research, 174, 24-33.

Drescher, M. S., Reinert, D. J., Denardin, J. E., Gubiani, P. I., Faganello, A., Silva, B. R. D., \& Zardin, M. C. (2017). Fertilizer shanks to promote soil decompaction in the seeding operation. Ciência Rural, 47(2), 1-8. 
Eduardo, E. N., Carvalho, D. F. D., Machado, R. L., Soares, P. F. C., \& Almeida, W. S. D. (2013). Erodibilidade, fatores cobertura e manejo e práticas conservacionistas em Argissolo Vermelho-Amarelo, sob condições de chuva natural. Revista Brasileira de Ciência do Solo, $37,796-803$.

EMBRAPA. Empresa Brasileira de Pesquisa Agropecuária. Sistema brasileiro de classificação de solos. Centro Nacional de Pesquisa de Solos. Brasília/DF: EMBRAPA CNPS, 2018. $5^{\mathrm{a}}$ Ed. 532p.

Gao, X., Xie, Y., Liu, G., Liu, B., \& Duan, X. (2015). Effects of soil erosion on soybean yield as estimated by simulating gradually eroded soil profiles. Soil and Tillage Research, 145, 126-134.

Gubiani, P. I., Lier, Q. D. J. V., Drescher, M. S., Mezzomo, H. C., \& Veiga, C. M. C. (2015). Relação entre densidade do solo e conteúdo de água em repetidos ciclos de contração e expansão em um Latossolo. Revista Brasileira de Ciência do Solo, 39, 100-108.

Gvozdenovich, J. J., Pérez Bidegain, M., Novelli, L. E., \& Barbagelata, P. A. (2017). ¿ Puede WEPP mejorar la predicción de la erosión de suelos respecto a USLE?. Ciencia del suelo, 35(2), 259-272.

Keesstra, S., Pereira, P., Novara, A., Brevik, E. C., Azorin-Molina, C., Parras-Alcántara, L., ... \& Cerdà, A. (2016). Effects of soil management techniques on soil water erosion in apricot orchards. Science of the Total Environment, 551, 357-366.

Mazurana, M., Levien, R., Inda, A. V., Conte, O., Bressani, L. A., \& Müller, J. (2017). Soil susceptibility to compaction under use conditions in southern Brazil. Ciência e Agrotecnologia, 41, 60-71.

Mello, C. R. D., Norton, L. D., Pinto, L. C., Beskow, S., \& Curi, N. (2016). Agricultural watershed modeling: a review for hydrology and soil erosion processes. Ciência e Agrotecnologia, 40, 7-25.

Miguel, P., Dalmolin, R. S. D., Pedron, F. D. A., Moura-Bueno, J. M., \& Tiecher, T. (2014). Identificação de fontes de produção de sedimentos em uma bacia hidrográfica de encosta. Revista Brasileira de Ciência do Solo, 38, 585-598.

Nascimento, V. D., Arf, O., Tarsitano, M. A. A., Garcia, N. F. S., Penteado, M. D. S., \& Sabundjian, M. T. (2016). Economic analysis of rice and common bean production in succession to green manure crops and mecahnical soil decompression in Brazilian Savannah 1. Revista Ceres, 63, 315-322.

Oliveira, L. C. D., Bertol, I., Barbosa, F. T., Campos, M. L., \& Mecabô, J. (2015). Perdas de solo, água e nutrientes por erosão hídrica em uma estrada florestal na Serra Catarinense. Ciência Florestal, 25, 655-665.

Oliveira, D., Lima, R. P. D., \& Verburg, E. E. J. (2015). Qualidade física do solo sob diferentes sistemas de manejo e aplicação de dejeto líquido suíno. Revista Brasileira de Engenharia Agrícola e Ambiental, 19, 280-285.

Olivetti, D., Mincato, R. L., Ayer, J. E. B., Silva, M. L. N., \& Curi, N. (2015). Spatial and temporal modeling of water erosion in dystrophic red latosol (oxisol) used for farming and cattle raising activities in a sub-basin in the South of Minas Gerais. Ciência e Agrotecnologia, $39,58-67$.

Ouyang, W., Wu, Y., Hao, Z., Zhang, Q., Bu, Q., \& Gao, X. (2018). Combined impacts of land use and soil property changes on soil erosion in a mollisol area under long-term agricultural development. Science of the total environment, 613, 798-809.

Pandey, A., Himanshu, S. K., Mishra, S. K., \& Singh, V. P. (2016). Physically based soil erosion and sediment yield models revisited. Catena, 147, 595-620.

Pereira, A. S., Shitsuka, D. M., Parreira, F. J., \& Shitsuka, R. (2018). Metodologia da pesquisa científica. [free e-book]. Santa Maria/RS. Ed. UAB/NTE/UFSM.

Prosdocimi, M., Jordán, A., Tarolli, P., Keesstra, S., Novara, A., \& Cerdà, A. (2016). The immediate effectiveness of barley straw mulch in reducing soil erodibility and surface runoff generation in Mediterranean vineyards. Science of the Total Environment, 547, 323-330.

Ramos, J. C., Bertol, I., Barbosa, F. T., Marioti, J., \& Werner, R. D. S. (2014). Influência das condições de superfície e do cultivo do solo na erosão hídrica em um Cambissolo Húmico. Revista Brasileira de Ciência do Solo, 38, 1587-1600.

Rodrigues, V. A., Sánchez-Román, R. M., Tarjuelo, J. M., Sartori, M. M. P., \& Canales, A. R. (2015). Avaliação do escoamento e interceptação da água das chuvas. Irriga, 1(1), 01-13.

Silva, A. A., \& Castro, S. S. D. (2015). Indicadores macro e micromorfológicos da qualidade física de um Latossolo Vermelho cultivado com cana-deaçúcar. Mercator (Fortaleza), 14, 169-185.

Statsoft, INC. (2011). Statistica (data analysis software system), version 10

R Core Team. (2015). R: A language and environment for statistical computing [Internet]. Vienna, Austria: R Foundation for Statistical Computing.

Ramos, J. C., Bertol, I., Barbosa, F. T., Marioti, J., \& Werner, R. D. S. (2014). Influência das condições de superfície e do cultivo do solo na erosão hídrica em um Cambissolo Húmico. Revista Brasileira de Ciência do Solo, 38, 1587-1600.

Vanwalleghem, T., Gómez, J. A., Amate, J. I., de Molina, M. G., Vanderlinden, K., Guzmán, G., ... \& Giráldez, J. V. (2017). Impact of historical land use and soil management change on soil erosion and agricultural sustainability during the Anthropocene. Anthropocene, 17, 13-29.

Volk, L. B. D. S., \& Cogo, N. P. (2014). Erosão hídrica, em três momentos da cultura do milho, influenciada por métodos de preparo do solo e semeadura. Revista Brasileira de Ciência do Solo, 38, 565-574. 